\title{
Micron poly-L-lactide fibers fabricated by melt direct writing
}

\author{
Huichao Liu ${ }^{a}$, Jingxin Kang ${ }^{b}$, Yong Liuc \\ College of Mechanical and Electric Engineering, Beijing University of Chemical Technology, Beijing \\ 100029, China
}

a907295059@qq.com, bkangjx@mail.buct.edu.cn, cyongsd@iccas.ac.cn

Keywords: poly-L-lactide (PLLA); three-dimensional (3D) print; a needle shaped collector.

Abstract:Through three-dimensional (3D) printing pen methods on the high-voltage electrostatic field with a needle shaped collector, the refined poly-L-lactide (PLA) finished product has been achieved. Through orthogonal experimental methods, We found that optimal conditions at $180^{\circ} \mathrm{C}$, a nozzle-to-needle shaped collector of $7 \mathrm{~mm}$, and applied voltage at $45 \mathrm{kV}$ with narrow variance distribution produce an average silk diameter reaching $517.1 \pm 24.9 \mu \mathrm{m}$. 3D printed in a high voltage electrostatic field fibers are 0.5 times thinner than direct 3D printed fibers. Four polymer patterns have been precisely printed. It can be used to make ordered micron fiber pattern, such as the micro sensor, air ultrafiltration membrane, Proton exchange membrane. In this report, the morphology, structural changes of the fibers are characterized.

\section{Introduction}

PLA is a degradable aliphatic polyester, which was discovered in 1932 by Carothers first in DuPont [1,2].The process of producing PLA creates less pollution and the processing temperature of PLA is controlled in $170 \sim 230^{\circ} \mathrm{C}[3,4]$. PLA has a series of excellent properties such as biocompatibility, biodegradability, mechanical properties and transparency [5-7]. Therefore, PLA is widely used in the field of 3D printing. In the experiments, a direct-current (DC) high voltage power supply is added to the 3D printing pen, to achieve the purpose of refinement of fiber diameter. The results showed that this method can control the diameter of fibers. In this report, the morphology, structural changes of the fibers are characterized. This method of precise control of PLA fiber paths and diameter is available for non-toxic air ultrafiltration membrane fabrication.

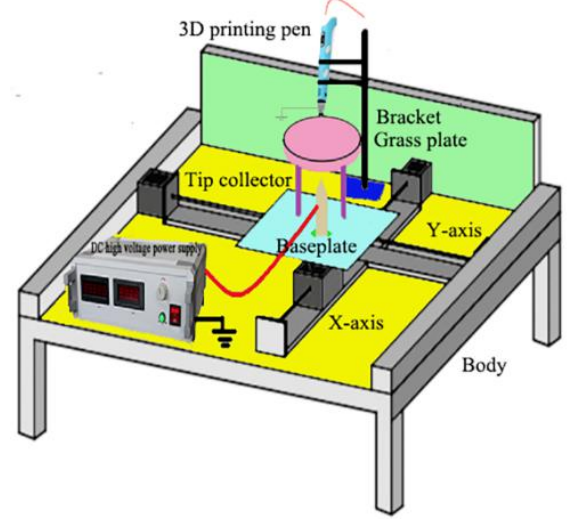

Figure 1. Schematic diagram of a 3D printing pen on the high-voltage electrostatic field.

\section{Experiments about 3D printing pen on high-voltage electrostatic field}

Materials and Methods. PLA and the 3D printing pen was purchased from AOD (China). PLA was used as the melt for DSC analysis, the range of heating temperature ranged from 25 to 200 centigrade and the heating rate is $10^{\circ} \mathrm{C} / \mathrm{min}$ in the nitrogen atmosphere. The schematic diagram of the experiments about 3D printing pen on the high-voltage electrostatic field was shown in Figure 1. The self-made needle shaped collector was a $0.82 \mathrm{~mm}$ in diameter. The DC high voltage power 
supply was purchased from Dong Wen (China).The stepper driver(ZD-6209-V2C) and the servo controller (DY-IS) was purchased from Times Brilliant. The needle shaped collector was connected to the positive high-voltage power-supply terminal, and the nozzle, covered with aluminum foil, was grounded. The needle shaped collector's movement was controlled by the X-and Y-axis. Samples were also prepared using a 3D printing pen without the high-voltage electrostatic field for comparison.

Characterization and Measurement. The morphology of the obtained fibers was photographed by a camera. The diameters of the fibers were measured by micrometer. The average fiber diameter and its standard deviation were calculated from the measured data using Image $\mathrm{J}$ software. The productivity was calculated under different conditions by measuring the qualities of the samples produced in 1 and a half minutes. Differential scanning calorimetry (DSC, PerkinElmer Pyris 1) was employed to analyze the crystallization behavior of the resulting fibers, with the samples being heated from $0^{\circ} \mathrm{C}$ to $1700{ }^{\circ} \mathrm{C}$ at $10^{\circ} \mathrm{C} / \mathrm{min}$ under a nitrogen atmosphere $(20 \mathrm{~mL} / \mathrm{min})$.

\section{Results and discussion}

The optimum conditions. In the present study, an orthogonal design table $\mathrm{L}_{17}(4){ }^{5}$ were designed using the software, Orthogonal Designing Assistant II (Share top Software Studio, v3.1). Each orthogonal table has its own mark denoted as $\mathrm{L}_{\mathrm{n}}(\mathrm{t})^{\mathrm{c}}$, $\mathrm{L}$ represents the orthogonal table, the number lower right corner of $\mathrm{L}$ is the total number of experiments, $t$ is the number of levels of each factor, the superscript (c), representing the maximum allowed a number of factors. The average diameter and standard deviation (SD) of average diameter were the evaluation indices of the orthogonal experiment in this study. The factors of the cylinder temperature, the nozzle- needle shaped collector distance, applied voltage and the flow rate (called here after temperature, distance, voltage, and speed) labeled as A, B, C, and D were investigated. The average diameters and SD of average diameter are shown in Table 1. Through orthogonal experimental methods, We found that optimal conditions at $180^{\circ} \mathrm{C}$, a nozzle-to-needle shaped collector of $7 \mathrm{~mm}$, and applied voltage at $45 \mathrm{kV}$ with narrow variance distribution produce an average silk diameter reaching $517.1 \pm 24.9 \mu \mathrm{m}$. But there are the following defects.There is a fiber accumulation in the corner, which can only be improved by reducing the speed at the turning point [8], such as Figure 2. a. As can be seen from Figures 2.a, when the heating temperature reaches $210^{\circ} \mathbf{C}$, the viscosity is too large to cause the fluid to fall too fast and can not form the fiber. Four polymer patterns have been precisely printed in Figure2.b. The higher the DC voltage, the finer the fiber, because the greater the voltage, the greater the electrostatic pull on the fiber [9]. If the voltage is too high, it will break into the dielectric plates collectors, resulting in a voltage drop. The influence of temperature and needle-collector distance on the fiber diameter is not obvious, but the temperature can not be above $210{ }^{\circ} \mathrm{C}$. The greater the feed rate, the thicker the fiber, because the fiber falls faster, and the time it takes to stretch the fibers is relatively short[10]. And the feed speed can not be too fast, which will lead to melting speed can not keep up. 
Table 1. Results and Analysis of Orthogonal $\mathrm{L}_{17}(4){ }^{5}$ Experimental Design;

\begin{tabular}{lllllll}
\hline Experiment NO. & \multicolumn{5}{c}{ Factors } & \multicolumn{2}{c}{ Results } \\
\cline { 2 - 7 } & $\mathrm{A}\left[{ }^{\circ} \mathrm{C}\right]$ & $\mathrm{B}[\mathrm{mm}]$ & $\mathrm{C}[\mathrm{kV}]$ & $\mathrm{D}[\mathrm{mm} / \mathrm{s}]$ & Average diameter[mm] & SD of average Diameter \\
\hline 1 & 180 & 6 & 30 & 20 & 0.5714 & 0.0352 \\
2 & 180 & 6 & 35 & 20 & 0.5628 & 0.031 \\
3 & 180 & 6 & 40 & 20 & 0.5542 & 0.0287 \\
4 & 180 & 6 & 45 & 20 & 0.55 & 0.0176 \\
5 & 180 & 5 & 45 & 20 & 0.5314 & 0.028 \\
6 & 180 & 4 & 45 & 20 & 0.5286 & 0.0259 \\
7 & 180 & 7 & 45 & 20 & 0.5171 & 0.0249 \\
8 & 180 & 7 & 45 & 40 & 0.6542 & 0.0256 \\
9 & 180 & 7 & 45 & 60 & 0.7571 & 0.0271 \\
10 & 190 & 7 & 45 & 60 & 0.7686 & 0.0919 \\
11 & 200 & 7 & 45 & 60 & 0.6429 & 0.058 \\
12 & 210 & 7 & 45 & 60 & 0.9314 & 0.1287 \\
13 & 180 & 6 & 0 & 60 & 0.8171 & 0.056 \\
14 & 180 & 7 & 0 & 20 & 0.7914 & 0.0181 \\
15 & 190 & 7 & 0 & 60 & 0.83 & 0.022 \\
16 & 200 & 7 & 0 & 60 & 0.8957 & 0.1034 \\
17 & 210 & 7 & 0 & 60 & 1 & 0.1111 \\
\hline
\end{tabular}

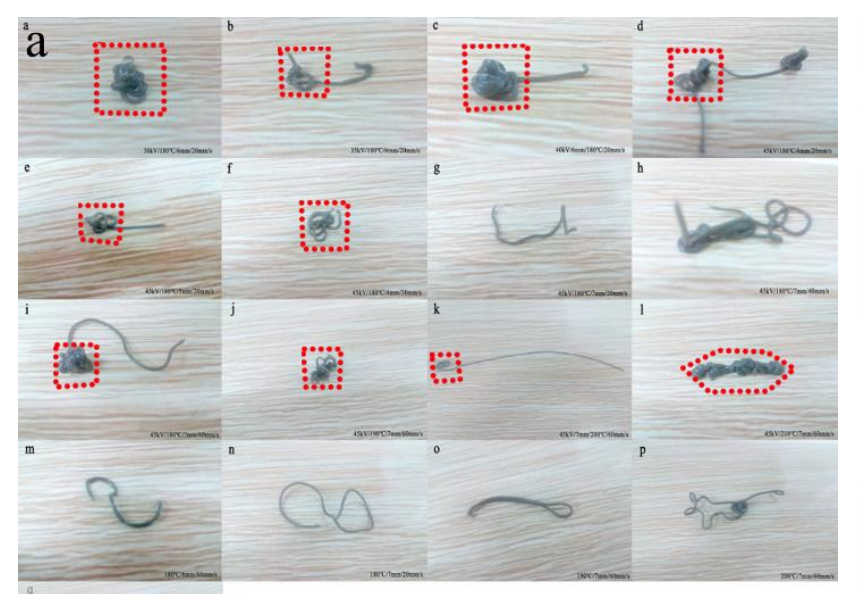

The figure corresponds to the experiments in table I: $\mathrm{a}-1, \mathrm{~b}-2$ $\mathrm{c}-3$, d-4, e-5, f-6, g-7, h-8, i-9, j-10, k-11, 1-12, m-13, n-14, $0-15, \mathrm{p}-16, \mathrm{q}-17$
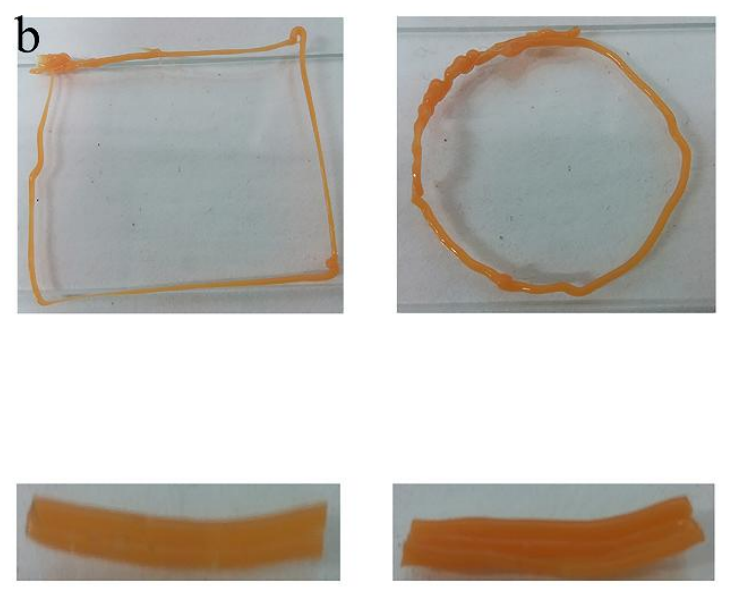

Average diameter $=17.61 \pm 0.55 \mu \mathrm{m}$.

$45 \mathrm{kV} / 180^{\circ} \mathrm{C} / 6 \mathrm{~mm} / 20 \mathrm{~mm} / \mathrm{s}$

Figure 2. The photos of (a) fibers written by a 3D printing pen; (b) multiple patterns printed by 3D printing pen on high-voltage electrostatic field. 

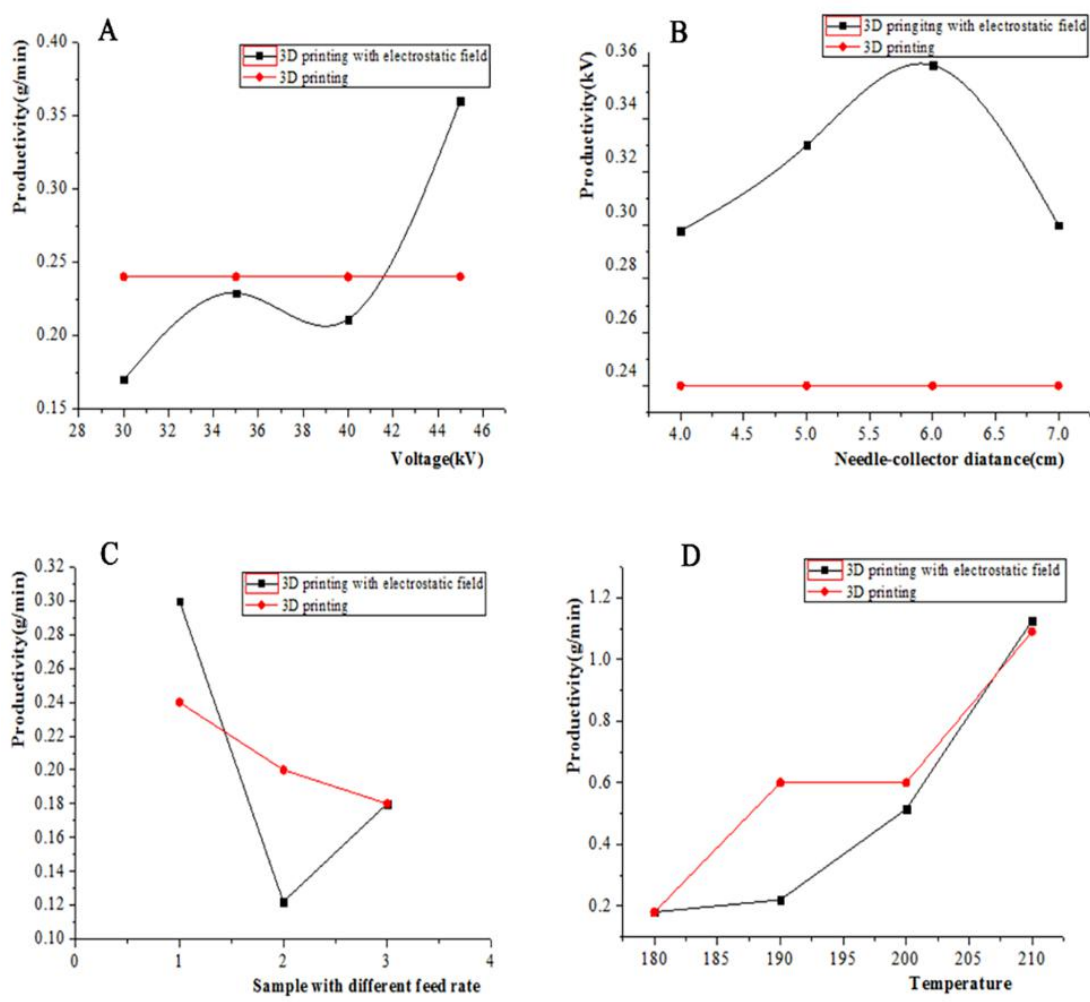

Figure 3. The law diagram of fiber productivity versus voltage, distance, feed rate, and temperature parameters.

The highest productivity. At the same time, the quality output per hour of the fiber under each condition are calculated. The law diagram of fiber productivity versus voltage, distance, feed rate, and temperature parameters is plotted in Figure 3. Only the effect of temperature on fiber productivity is obvious. This is because the greater the temperature, the greater the viscosity, the faster the fiber falls, the higher the productivity, as shown in Figure 3.D. This is consistent with the results and regularities of electrospinning process simulated by Wang [11]. When the heating temperature reaches $210{ }^{\circ} \mathrm{C}$, the voltage reaches $45 \mathrm{kV}$, the distance is $7 \mathrm{~mm}$ and the speed is $60 \mathrm{~mm} / \mathrm{s}$, the highest productivity of the fiber is $1.125 \mathrm{~g} / \mathrm{min}$. When the voltage, the feed rate, the distance and the temperature is the maximum at the same time the production rate is maximum. This is because under this condition, the fiber is subjected to the maximum electric field force for the longest time and the maximum viscosity, so the speed of spinning is the fastest.

Crystallization behavior about 3D printing pen parameters. In order to verify the above results, DSC was carried out to further investigate the influence of High voltage electrostatic fields on the internal structure of PLA fibers, as shown in Figure 4. When polymers are heated to over $T_{g}$, molecules change into low energy states, forming newly condensed tangles and releasing heat. Thus, an exothermic peak appears on the curve. Polymers form endothermic peaks near $\mathrm{T}_{\mathrm{m}}$. The crystallinity of the polymer can be obtained by measuring the heat of fusion of the sample divided by the reference value. It can be seen that the addition of the high voltage electrostatic field will destroys the regularity of the molecular chain, which reduce the crystallinity of the fibers[12]. 

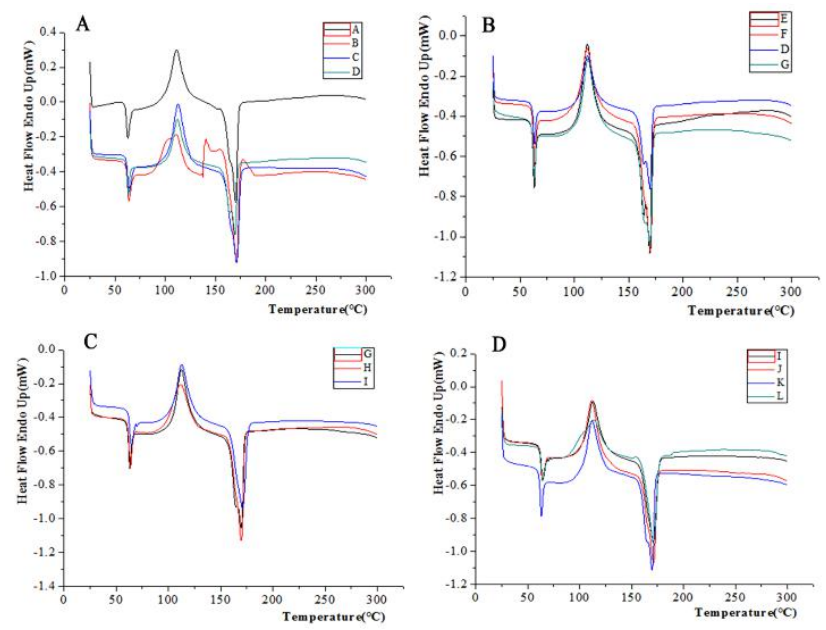

Figure 4. Diagram of fiber productivity versus voltage, distance, feed rate, and temperature parameters.

\section{Conclusions}

3D printing pen on the high-voltage electrostatic field were experimentally demonstrated to reduce the diameter of fibers. As the voltage increases, both the diameter and the crystallinity of the fibers decrease in a certain range. 3D printed fibers in a high voltage electrostatic field are 0.5 times thinner than the 3D printed fibers. But there are some protrusions in the fiber and on the surface. This may be related to the concentration, jet stretching, and solidification. To sum up, adding extra high-voltage electric field to 3D printer and collectors with needle tip are both very potential methods, which deserve further study.

\section{References}

[1] G.L. Siparsky, K.J. Voorhees. J. Environ. Polym. Degrad. 31-41 (1998), p. 6.

[2] N. Phothapaeree, N. Jarusaksakul and A. Somwangthanaroj. J. Sci. Technol. 253-260 (2017).

[3] X. Sun. Bio based chemicals and polymers and their applications for adhesives, plastics, and composites, 2013. ASABE. Int. Meeting. 21-24 (2013), p.21.

[4] H. J. Zheng, Y. R. Wang, and Z. W. Zhao. Adv. Mater. Res. 550-553 (2012) p.552.

[5] W. Fu. J. Reinf. Plast. Comp. 2034-2045 (2014) p.2035.

[6] F. B. Ali, D. J. Kang and M. P. Kim. Polym. Int. 1620-1626 (2014) p.1623.

[7] Z. Z. Han, Y. C. Zhang, and W. M. Yang. Key. Eng. Mater. 68-72 (2017), p.71.

[8] Y. L. Qi, J. T. Yu, Heilongjiang. Sci. Technol. Inf. 111 (2016) p.111.

[9] Z. F. Li. J. Jilin. Inst. Chem. Technol. 19-22 (2008), p.22.

[10] C. Yao, X. S. Li and S.B. Wu. Chin. Mater. Symp. (China, 2004).

[11] X. Wang, Y. Liu and C. Zhang. J. Nanosci. Nanotechnol. 4680-4685 (2013).

[12] G. Xie, Z. Chen and S. Ramakrishna. J. Appl. Polym.Sci. (2015), p. 132. 